\title{
Estimating VDT Mental Fatigue Using Multichannel Linear Descriptors and KPCA-HMM
}

\author{
Chong Zhang, Chongxun Zheng, Xiaolin Yu, and Yi Ouyang \\ Key Laboratory of Biomedical Information Engineering of Education Ministry, School of Life Science and Technology, \\ Xi'an Jiaotong University, 710049 Xi'an, China \\ Correspondence should be addressed to Chongxun Zheng, cxzheng@mail.xjtu.edu.cn
}

Received 28 September 2007; Revised 23 December 2007; Accepted 21 February 2008

Recommended by Sergios Theodoridis

The impacts of prolonged visual display terminal (VDT) work on central nervous system and autonomic nervous system are observed and analyzed based on electroencephalogram (EEG) and heart rate variability (HRV). Power spectral indices of HRV, the P300 components based on visual oddball task, and multichannel linear descriptors of EEG are combined to estimate the change of mental fatigue. The results show that long-term VDT work induces the mental fatigue. The power spectral of HRV, the P300 components, and multichannel linear descriptors of EEG are correlated with mental fatigue level. The cognitive information processing would come down after long-term VDT work. Moreover, the multichannel linear descriptors of EEG can effectively reflect the changes of $\theta, \alpha$, and $\beta$ waves and may be used as the indices of the mental fatigue level. The kernel principal component analysis (KPCA) and hidden Markov model (HMM) are combined to differentiate two mental fatigue states. The investigation suggests that the joint KPCA-HMM method can effectively reduce the dimensions of the feature vectors, accelerate the classification speed, and improve the accuracy of mental fatigue to achieve the maximum $88 \%$. Hence KPCA-HMM could be a promising model for the estimation of mental fatigue.

Copyright (c) 2008 Chong Zhang et al. This is an open access article distributed under the Creative Commons Attribution License, which permits unrestricted use, distribution, and reproduction in any medium, provided the original work is properly cited.

\section{INTRODUCTION}

Mental fatigue is a common physiological phenomenon. Especially in highly demanding visual display terminal (VDT) work,people may become fatigued and suffer some experience difficulties for maintaining task performance at an adequate level [1]. In industry, many incidents and accidents are related to VDT mental fatigue as the result of sustained performance [2]. It is important to cope with mental fatigue so that the workers do not harm their health. Therefore, the management of fatigue is very important not only for enhancing productivity, but also for protecting occupational health.

To date, many methods have been proposed to estimate the mental fatigue. A large number of previous studies use behavioural indices or subjective measures such as reaction time, error ratio, or subjective scales. However, these measures have some limitations, for instance, they cannot provide moment-to-moment fluctuations of mental fatigue. Moreover, the results may be affected by the subjects' cogni- tive ability, mood, and anxiety levels [3-5]. A recent tendency in ergonomic research is to choose more objective measures to assess the mental fatigue state. These approaches focus on measuring physiological changes of people, such as the electrooculogram (EOG), respiratory signals, heart beat rate, skin electric potential, and particularly, electroencephalographic (EEG) activities as a means of detecting the mental fatigue states. Some scholars reported that performing monotonous tasks was related to the increase of the $0.1 \mathrm{~Hz}$ component in the heart rate variability (HRV) $[6,7]$. Although numerous physiological indicators were available to describe an individual's mental fatigue state, the EEG signals might be the most promising, predictive, and reliable one $[8,9]$. The EEG was widely regarded as the physiological "gold standard" for the assessment of mental fatigue. There were several EEG studies related to mental fatigue in the past. Some studies reported EEG spectral changes as alertness declines. For example, the proportion of low-frequency EEG waves, such as $\theta$ and $\alpha$ rhythms, might increase while higherfrequency waves, such as $\beta$ rhythms might decrease [10-12]. 
Other studies explored the links between fatigue and changes in event-related potential (ERP) components. Mental fatigue was found to produce a decrease in P300 amplitude while latency increases [13-15].

However, mental fatigue is a complex phenomenon, which is influenced by the environment, the state of health, vitality, and the capability of recovery. Single physiological parameter cannot evaluate mental fatigue well. It would preferably need to consider more objective physiological measures. Thus, several techniques need to be combined to estimate the state of mental fatigue. Lorist et al. used ERP and mood questionnaires to assess the effects of mental fatigue [16]. Lal and Craig combined EEG activity, heart rate with psychological measures such as anxiety, tension, and fatigue levels to investigate the impacts of driving fatigue on subjects [5]. These previous studies showed that the EEG, electrocardiogram (ECG) activity, and psychological factors were associated with mental fatigue.

In this paper, the subjective self-reporting measures are utilized to verify that long-term VDT work would induce the mental fatigue to the subjects. Then neurophysiological indicators such as the P300 amplitude and latency based on visual oddball task and multichannel linear descriptors in five frequency bands of EEG are further used to investigate the effect of prolonged VDT work on central nervous system, the power spectral parameters of HRV are applied to explore the effect on autonomic nervous system. Finally, kernel principal component analysis (KPCA) and hidden Markov model (HMM) are combined to measure momentto-moment mental fatigue changes. Compared with previous studies, the presented comprehensive methods would make the mental fatigue estimation much reliable and accuratecy since many psychological and physiological parameters are considered.

\section{MATERIALS AND METHODS}

\subsection{Subjects}

Fifty male right-dominated graduate students, between 20 and 27 years old $(\mathrm{M}=23.0$ years, $\mathrm{SD}=1.6)$, participated in this study. Personal data (handedness, past medical history, medical family history, etc.) were acquired with a standardized interview before EEG recordings. All subjects were in good health. None of them reported on any cardiovascular disease or neurological disorders in the past or had taken any drugs known to affect the EEG. Subjects did not work night shifts and had normal sleep time. All of them were accustomed to use the computer mouse and agreed to join the study.

\subsection{Experiment design}

Participants were comfortably seated facing a CRT video monitor at about $50 \mathrm{~cm}$ far. The illumination on the CRT was about $300 \mathrm{~lx}$. The experimental tasks were three types of simple VDT tasks. The first type of task was a vigilance task. Three random numbers displayed at the same time on the
CRT screen and changed once every second randomly. The subjects were asked to click the right mouse button promptly, as three different odd numbers, such as 1, 7, 9, appeared. Sixteen subjects participated in this experiment. The second type of task was the addition and subtraction arithmetic calculation of four one-digit numbers. They were displayed on a computer monitor continuously until the subject responded. The participants solved the problems firstly, and then decided whether the result was less than, equal to, or greater than the target sum provided. Sixteen subjects participated in this experiment. The third type of task was a simple switch task. A white square, subdivided into four subsquares, was displayed continuously at the screen center. Stimulus images were presented in turn, and the image was starting from the upper left subsquare with clockwise fashion. The stimulus images were numbered from zero to nine randomly. The color of the stimulus images was red or blue randomly. Then the subjects should pushed the left or right mouse button related to the image color, respectively, when the stimulus image appeared in either of two upper subsquares, or related to the odd or even number identity if the stimulus appeared in either of two lower subsquares. Eighteen subjects participated in this experiment. All subjects performed the VDT task until either they quitted from exhaustion or two hours elapsed. The response time and the number of error trials, if any, were recorded.

Subjects were required to abstain from alcohol and caffeine-containing substances 24 hours before the experiment. Subjects were told the study was aimed at investigating the neural correlates of cognitive control, they were unaware the study was about mental fatigue. To avoid the influence of circadian fluctuations on subjects, the experiments were scheduled to be at the same time session. The experimental session started about 8:00 and lasted for 3.5 to 4 hours. No any clock and watch in the laboratory. They had no knowledge about experimental duration.

Subjects were seated in a dimly lit, sound-attenuated, electrically shielded room. Before starting the experiment, the subjects completed a brief demographic questionnaire (age, handedness, hours of sleep, etc.), and ensured that the instructions were understood. First, the psychological selfreport measures of sleepiness and fatigue were conducted, and the ERPs were measured. Subjective sleepiness was assessed by means of the Stanford sleepiness scale and the Karolinska sleepiness scale, and subjective fatigue was measured with the help of the Samn-Perelli checklist, Li's subjective fatigue scale and Borg's CR-10 scale [7, 17-20]. Subsequently, the subjects were required to simply relax and try to think of nothing in particular, and recorded the EEG and ECG in the eyes-closed resting state for five minutes before starting the experimental session. They then performed the VDT task either until two hours elapsed or until volitional exhaustion occurred. Subjects were instructed to respond as quickly as possible, maintaining a high level of accuracy. EEG and ECG recordings were conducted immediately after the completion of the VDT task. The same psychological rating and ERPs measurement were also carried out at two epochs: pretask, that was before task; posttask, that was immediately after task. 
The ERP was recorded when the subject was doing the visual oddball task. The red ball and green ball images were used for the visual stimulations. The probabilities of appearance of green and red balls were 0.8 and 0.2 , respectively. The subject was ordered to respond to the rare stimuli with a response switch as fast as possible. The stimulus interval was $1000 \pm 200$ milliseconds. The 160 milliseconds EEG data before stimulus onset and the 640 milliseconds EEG data after stimulus onset were used for averaging ERPs. And then the peak latency and the corresponding amplitude for the P300 components could be well obtained.

\subsection{Data acquisition}

EEGs were recorded by a Neuroscan 32 channel system (Neuroscan, El Paso, Tex, USA) with international 10-20 lead systems. Fp2, Fp1, F4, F3, A2, A1, C4, C3, P4, P3, $\mathrm{Fz}, \mathrm{Cz}$, and $\mathrm{Pz}$ leads were used with $\mathrm{Ag} / \mathrm{AgCl}$ electrodes. Recordings were referenced to linked-mastoids. Two additional bipolar pairs of electrodes were placed to record horizontal and vertical EOG. ECG was measured with three disposable electrodes. Two electrodes were attached on the left and right sides of frontal lower ribs. The grounding electrode was placed on the sternum. Skin impedance was below $5 \mathrm{k} \Omega$ on all electrodes. Physiological signals were filtered by band pass filter with bandwidth from 0.01 to $100 \mathrm{~Hz}$. The signal was sampled at $500 \mathrm{~Hz}$ and digitized at 16 bits. Eye movement contamination was removed by adaptive filtering methods.

\subsection{Power spectral of HRV}

Power spectrum analysis of HRV is a sensitive and noninvasive technique to quantify the autonomic control over the cardiac cycle [21]. It usually uses high-frequency (HF: $0.15-0.40 \mathrm{~Hz}$ ) power as an index of parasympathetic activity and the low-frequency (LF: $0.04-0.15 \mathrm{~Hz}$ ) power as an index of sympathetic and parasympathetic activity [22]. Thus, The $\mathrm{LF} / \mathrm{HF}$ ratio is considered to mirror sympathovagal balance or to reflect the sympathetic modulations $[23,24]$.

The detection of $\mathrm{R}$ waves is done by the wavelet transform-based algorithm. Then 5-minute raw R-R interval sequence is interpolated at 1-second intervals by linear interpolation. From the interpolated RR tachogram, the power spectrum of HRV is estimated from $256 \mathrm{R}-\mathrm{R}$ intervals of the heart beat by using an autoregressive (AR) model (order 16). When $\mathrm{R}-\mathrm{R}$ spectrums are investigated, it is observed that a considerable amount of energy is in the very low-frequency (VLF) range $(<0.03 \mathrm{~Hz})$. VLF oscillations are much less defined, but suggest to be related with thermoregulation. Therefore, to prevent these oscillations from masking other frequency ranges $(<0.03 \mathrm{~Hz})$, they are filtered from the tachograms by using wavelet filter before modeling.

For spectral analysis, the total power (TP: 0-0.4 Hz), LF power, HF power, and LF/HF ratio are calculated. Spectrum components are expressed both in absolute unit $\left(\mathrm{AU}, \mathrm{ms}^{2}\right.$ ) and normalized unit (NU). The normalized value of equa- tion of the LF power is represented as

$$
\text { LF power }(\mathrm{NU})=\frac{\mathrm{LF} \text { power }}{\mathrm{TP} \text { power }-\mathrm{VLF} \text { power }} \text {. }
$$

\subsection{Feature extraction based on multichannel linear descriptors}

Wackermann proposed a $\Sigma-\Phi-\Omega$ system for describing the comprehensive global brain macrostate [25]. Let us consider $N$ EEG samples in the observed time window at $K$ electrodes to construct the voltage vectors $\left\{u_{1}, \ldots, u_{N}\right\}$, where each $u_{i}(i=1, \ldots, N)$ corresponds to the statevector representing the spatial distribution of EEG voltage over the scalp at the $i$ th sample. The data are assumed to have been already centered to zero mean and transformed to the average reference $[25,26]$. Then $\Omega, \Phi$, and $\Sigma$ can be calculated as follows [26]:

$$
\begin{aligned}
m_{0} & =\frac{1}{N} \sum_{i}\left\|u_{i}\right\|^{2}, \\
m_{1} & =\frac{1}{N} \sum_{i}\left\|\frac{\Delta u_{i}}{\Delta t}\right\|^{2}, \quad \text { where } \Delta u_{i}=u_{i}-u_{i-1}, \\
\sum & =\sqrt{\frac{m_{0}}{K}} \\
\Phi & =\frac{1}{2 \pi} \sqrt{\frac{m_{1}}{m_{0}}} .
\end{aligned}
$$

The covariance matrix is constructed as:

$$
C=\frac{1}{N} \sum_{n} u_{n} u_{n}^{T}
$$

The eigenvalues $\lambda_{1} \cdots \lambda_{K}$ of matrix $C$ is calculated, then $\Omega$ complexity can be obtained:

$$
\log \Omega=-\sum_{i} \lambda_{i}^{\prime} \log \lambda_{i}^{\prime}
$$

where $\lambda_{i}^{\prime}$ is the normalized eigenvalue.

In the $\Sigma-\Phi-\Omega$ system, $K$-dimensional voltage vectors constructed from the simultaneous EEG measurements over $K$ electrodes with time varying are regarded as the trajectories in the $K$-dimensional state space. By the three linear descriptors, the physical properties of the EEG trajectory and then the brain macrostates are characterized. $\Phi$ reflects mean frequency of the corresponding field changes; $\Omega$ measures the spatial complexity of the brain region, which decomposes the multichannel EEG data into spatial principal components and then quantifies the degree of synchrony between the distributed EEG by the extension along the principal axes; larger value of $\Omega$ corresponds to the low synchrony; $\Sigma$ reflects the corresponding regional field power; $\Phi$ characterizes the speed of regional field changes of the contralateral, ipsilateral, and mid-central regions, respectively. It can be seen that by EEG over the $K$ electrodes the three linear descriptors describes the different brain macorstate features of the interested brain regions. 
In this paper, after artifact detection and ocular correction, one-minute EEG data of each trial for each subject in the session of pretask and posttask are selected to be analyzed. The first 10 seconds EEG data is chosen as basic data segment and steps by one-second data. By shifting the data segment step-by-step for whole trial, 5100 data segments are obtained.

Wavelet packet analysis is performed to every EEG data segment. Daubechies 10 is adopted as the mother wavelet. After eight-octave wavelet packet decomposition, the EEG components of the following five frequency bands are obtained: total $(0.5-30 \mathrm{~Hz})$, delta $(0.5-3.5 \mathrm{~Hz}), \theta(4-7 \mathrm{~Hz}) \alpha(8-$ $12 \mathrm{~Hz})$, and $\beta(13-30 \mathrm{~Hz})$. Twelve electrode arrays formed by eleven electrodes are used, that is, prefrontal electrode arrays (Fp1-Fz, Fp2-Fz, and Fp1-Fz-Fp2), frontal electrode arrays (F3-Fz, F4-Fz, and F3-Fz-F4), central electrode arrays (C3$\mathrm{Cz}, \mathrm{C} 4-\mathrm{Cz}$, and $\mathrm{C} 3-\mathrm{Cz}-\mathrm{C} 4)$ and parietal electrode arrays (P3$\mathrm{Pz}, \mathrm{P} 4-\mathrm{Pz}$, and P3-Pz-P4). Then $\Omega, \Phi$, and $\Sigma$ of all EEG data segments are calculated for every electrode array in five frequency bands, respectively, and multichannel linear descriptor features of 180 dimensions for every EEG data segment are obtained.

\subsection{Reducing the dimensions of feature vectors in feature spaces based on kernel PCA}

Kernel PCA (KPCA) as a nonlinear feature extractor has been proven powerful as a preprocessing step for classification algorithms. It first maps the data into some feature space $\mathbf{F}$ via a function $\gamma$ (usually nonlinear) and then performs linear PCA (LPCA) on the mapped data. As the feature space F may be very high dimensional, KPCA employs Mercer kernels instead of carrying out the mapping $\gamma$ explicitly.

The feature vectors preprocessed by KPCA have lower size, and can improve the generalization and speed of classification in the next step. The Gaussian function is selected as the kernel function for KPCA algorithm.

\subsubsection{KPCA with centered data in feature spaces}

Consider a nonlinear mapping $\gamma: \tilde{x} \rightarrow x, \tilde{\mathbf{x}} \in \mathbf{R}^{s}$ is the input vector space and $\mathbf{x} \in \mathbf{F}$ is the vector feature space. Assume the vector be centered as follows:

$$
\sum_{k=1}^{l} \gamma\left(\widetilde{x}_{k}\right)=0
$$

The covariance matrix $\mathbf{E}$ in the feature vector space $\mathbf{F}$ is

$$
\mathbf{E}=\frac{1}{l} \sum_{k=1}^{l} \gamma\left(\tilde{x}_{k}\right) \gamma^{T}\left(\tilde{x}_{k}\right) .
$$

Like LPCA, one has to solve the eigenvalue for a KPCA problem in the feature vector space:

$$
\lambda \boldsymbol{v}=\mathbf{E} \boldsymbol{v} .
$$

The solution $v$ lies in the span of $\gamma\left(\tilde{x}_{1}\right), \gamma\left(\tilde{x}_{2}\right), \ldots, \gamma\left(\tilde{x}_{l}\right)$ and (7) is equivalent to

$$
\lambda\left\langle\gamma\left(\tilde{x}_{k}\right), \boldsymbol{v}\right\rangle=\left\langle\gamma\left(\tilde{x}_{k}\right), \mathbf{E} \boldsymbol{v}\right\rangle,
$$

and there exists coefficients $\left\{c_{i}\right\}$, such that

$$
\boldsymbol{v}=\sum_{i=1}^{l} c_{i} \gamma\left(\tilde{x}_{i}\right) .
$$

Define a $l \times l$ dot product matrix:

$$
[\widetilde{K}]_{i j}=\widetilde{K}\left(\tilde{\mathbf{x}}_{i}, \tilde{\mathbf{x}}_{j}\right)=\left\langle\gamma\left(\tilde{x}_{i}\right), \gamma\left(\tilde{x}_{j}\right)\right\rangle,
$$

where $\widetilde{K}\left(\widetilde{\mathbf{x}}_{i}, \widetilde{\mathbf{x}}_{j}\right)$ is a kernel function satisfying Mercer's condition [27], that is, if and only if for any $g(x)$ s.t. $\int g(x)^{2} d x$ is finite, then $\int \tilde{K}\left(\tilde{\mathbf{x}}_{i}, \tilde{\mathbf{x}}_{j}\right) g\left(\tilde{x}_{i}\right) g\left(\tilde{x}_{j}\right) d \tilde{x}_{i} d \tilde{x}_{j} \geq 0$.

Combining (7)-(10), we have the following formula:

$$
\lambda l \mathbf{c}=\tilde{\mathbf{K}} \mathbf{c}, \quad \mathbf{c}=\left[\begin{array}{llll}
c_{1} & c_{2} & \cdots & c_{l}
\end{array}\right]^{T} .
$$

The solutions $\left(\lambda_{k}, \mathbf{c}^{k}\right)$ need to be normalized [28] by

$$
\lambda_{k}\left\langle\mathbf{c}^{k}, \mathbf{c}^{k}\right\rangle=1 \text {. }
$$

\subsubsection{Kernel PCA with noncentered data in feature spaces}

With mentioned above, the assumption that the mapped data are centered in $\mathbf{F}$ space is necessary (see (13)). For any $\gamma$ and any set of observations $\widetilde{\mathbf{x}}_{1}, \widetilde{\mathbf{x}}_{2}, \ldots, \widetilde{\mathbf{x}}_{l}$, the vectors will be centered as follows:

$$
\bar{\gamma}\left(\tilde{x}_{k}\right)=\gamma\left(\tilde{x}_{k}\right)-\frac{1}{l} \sum_{k=1}^{l} \gamma\left(\tilde{x}_{k}\right) .
$$

Similarly, we can get the formulas as follows:

$$
\begin{aligned}
\bar{\lambda} l \overline{\mathbf{c}} & =\overline{\mathbf{K}} \overline{\mathbf{c}}, \\
\overline{\mathbf{K}} & =\widetilde{\mathbf{K}}-\mathbf{1}_{l} \widetilde{\mathbf{K}}-\widetilde{\mathbf{K}} \mathbf{1}_{l}+\mathbf{1}_{l} \widetilde{\mathbf{K}} \mathbf{1}_{l},
\end{aligned}
$$

where $\mathbf{1}_{i j}=1$ and $\left(\mathbf{1}_{N}\right)_{i j}=\mathbf{1} / l$ for all $i, j$. Let $\tilde{\mathbf{x}}$ be a test vector, then $q$ nonlinear principal components corresponding to $\bar{\phi}$ when $\lambda_{1} \geq \lambda_{2} \geq \cdots \geq \lambda_{N}$ can be obtained by

$$
\left\langle\overline{\mathbf{c}}^{k}, \tilde{\mathbf{x}}\right\rangle=\sum_{i=1}^{l} \bar{c}_{i}^{k} \bar{K}\left(\widetilde{\mathbf{x}}_{i}, \widetilde{\mathbf{x}}\right), \quad k=1,2, \ldots, q .
$$

In summary, the following steps are necessary to compute KPCA [29]:

(i) for a set of $s$-dimensional training set $\left\{\widetilde{\mathbf{x}}_{k}\right\}$, the kernel matrices $\widetilde{\mathbf{K}}$ and $\overline{\mathbf{K}}$ defined by (10) and (15) are computed, respectively;

(ii) solve the eigenvalue problem (14) and normalize $\overline{\mathbf{c}}^{k}$ such that $\left\langle\overline{\mathbf{c}}^{k}, \overline{\mathbf{c}}^{k}\right\rangle=1 / \bar{\lambda}_{k}$;

(iii) let $\lambda_{1} \geq \lambda_{2} \geq \cdots \geq \lambda_{N}$. For a test pattern $\tilde{\mathbf{x}}, q$ nonlinear components in feature vector space are extracted by (16). The dimensions of the test pattern $\tilde{\mathbf{x}}$ are reduced from $s$ to $q$.

\subsection{Classification using hidden Markov model (HMM)}

The HMM can be seen as a finite automaton, containing $s$ discrete states, emitting a feature vector at every time point 
depending on the current state. Each feature vector is modeled using $\mathrm{m}$ Gaussian mixtures per state. The transition probabilities between states are described using a transition matrix. During the training phase, the expectation maximization (EM) algorithm introduced by Dempster [30] is used to estimate the transition matrix and the Gaussian mixtures. Based on randomly selected values for the transition matrix and an initial estimation of the mixtures, the EM algorithm is performed. The estimation formulas guarantee a monotonic increase of the likelihood $P(\nu \mid \mathrm{HMM})$ until reaching a local or global maximum to end the training phase.

The Gaussian mixtures are approximated based on a $k$ means clustering of the feature vectors. The clustering is performed using the Euclidean distance, which necessarily needs feature vector components with a mean and variance within the same numerical range. The mean and variance of all feature vectors belonging to one cluster are then used to model the Gaussian mixtures with a diagonal covariance matrix. This modeling is feasible just for the noncorrelated feature vector components. In order to meet both requirements of normalized and not correlated data, the whitening transformation is performed. The original data $V=(\nu(1), \nu(2) \cdots v(T))$ of length $T$ is transformed into $\bar{V}=(\bar{\nu}(1), \bar{\nu}(2) \cdots \bar{\nu}(T))$ using the following:

$$
\bar{V}=\Phi \Delta^{1 / 2} V
$$

where $\Phi$ and $\Delta$ the eigenvector and eigenvector matrices, respectively, of the covariance matrix of $V$.

Two HMM's, one representing the norm state $\left(\mathrm{HMM}_{N}\right)$ and one representing the fatigue state $\left(\mathrm{HMM}_{F}\right)$ are trained by using the EEG data segments recorded during the corresponding mental fatigue states. The parameters of the models are estimated by the given training data and are then used to classify the same training data. Finally, $\mathrm{HMM}_{N}$ and $\mathrm{HMM}_{F}$ are estimated by using the correct classified trials. Classification of an unknown EEG data segment is based on a selection of the maximum single best path probability $P_{p}(\bar{V} \mid \mathrm{HMM})$ calculated via the Viterbi algorithm [31]. Calculating $P_{p}\left(\bar{V} \mid \mathrm{HMM}_{N}\right)$ and $P_{p}\left(\bar{V} \mid \mathrm{HMM}_{F}\right)$ for all EEG segments will result in a propagation of these probabilities, which allows us to make classification sample by sample.

Cross-validation is a commonly used standard test method to test the classification ability by using various combinations of the testing and training data sets $[32,33]$. A 5-fold cross-validation test is applied, in which 50 subjects' data are divided into five groups. We randomly select 40 subjects' data as the train sample set, and 10 subjects' data as testing sample set. Each of the five cross-validation test groups, therefore, has 1020 EEG data segments of ten subjects while their respective training segment includes the remaining 4080 EEG data segments of forty subjects' data. To classify the test vectors given by our 5-fold cross-validation scheme, the likelihood of them to belong to each of two HMM's is calculated. The one having more likelihood is assigned to that mental fatigue state.
Three measures, accuracy (Ac), specificity (Sp) and sensitivity (Se) are used to assess the performance of five classifiers [34]:

$$
\begin{aligned}
\text { Accuracy } & =\frac{\mathrm{TP}+\mathrm{TN}}{\mathrm{TP}+\mathrm{FP}+\mathrm{TN}+\mathrm{FN}} \times 100 \%, \\
\text { Specificity } & =\frac{\mathrm{TN}}{\mathrm{TN}+\mathrm{FP}} \times 100 \%, \\
\text { Sensitivity } & =\frac{\mathrm{TP}}{\mathrm{TP}+\mathrm{FN}} \times 100 \%,
\end{aligned}
$$

where TP is the number of true positives, TN is the number of true negatives, FP is false norm identifications, and FN is false fatigue identification. The specificity is defined as the ability of the classifier to correctly recognize a fatigue state. The sensitivity indicates the classifier's ability not to generate a false detection (normal state). Figure 1 shows the schematic diagram for KPCA-HMM.

\section{RESULTS}

\subsection{Subjective evaluation of mental fatigue}

The results of comparison of several subjective scores between two sessions are shown in Figure 2.

The self-report questionnaires reveals that subjects are not fatigue and sleepy before task and moderately to extremely fatigue and sleepy after task. Compared with the pretask, the subjective scores increase significantly $(P<.005)$ after the completion of the task, which indicates that continuous long-term VDT task leads to an increase in fatigue and sleepiness.

\subsection{Mental fatigue analysis based on power spectral of HRV}

Figure 3 presents the power spectral of HRV made on a particular subject in the sessions of pretask and posttask. It is obvious that LF power increases after task.

Mean values of heart rate (HR), TP power, HF power (AU and NU), LF power (AU and NU), and LF/HF ratio of HRV between the pretask and posttask periods are shown in Table 1.

Compared with the pretask, mean HR and HF power (NU) decrease $(P<.005)$, while LF power (AU and NU), $\mathrm{LF} / \mathrm{HF}$ ratio and TP power increase $(P<.005)$ after the task. However, HF power (AU) does not change significantly.

\subsection{ERP analysis based on visual oddball task}

Figure 4 shows theexamples of P300 waveform at electrode locations $\mathrm{Fz}, \mathrm{Cz}$, and $\mathrm{Pz}$ in the pretask and posttask.

The statistical analysis results of P300 amplitude and latency at electrode locations $\mathrm{Fz}, \mathrm{Cz}$, and $\mathrm{Pz}$ are shown in Figure 5.

Compared with the pretask, P300 amplitudes at electrode locations $\mathrm{Fz}, \mathrm{Cz}$, and $\mathrm{Pz}$ all decrease $(P<.005)$ after the task, while P300 latencies at electrode locations $\mathrm{Fz}, \mathrm{Cz}$, and $\mathrm{Pz}$ all increase $(P<.005)$ significantly. Moreover, the mean 


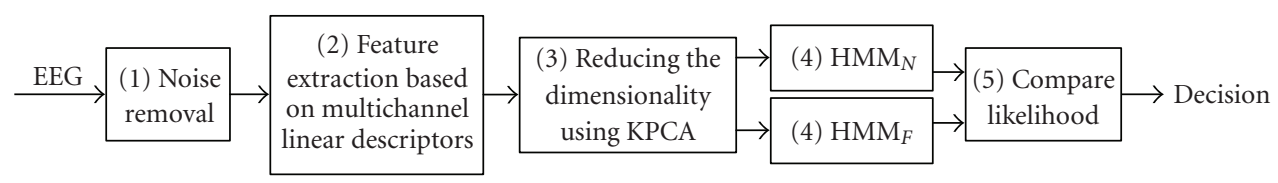

FIgURE 1: Schematic diagram for KPCA-HMM: (1) eye movement contamination is removed by adaptive filtering methods; (2) features of 180 dimensions are extracted using multichannel linear descriptors measure; (3) kernel PCA is used to reduce the dimensions of features; (4) two HMM's are trained, $\mathrm{HMM}_{N}$ corresponds to the norm state, and $\mathrm{HMM}_{F}$ corresponds to the fatigue state; and (5) the final mental state is decided by the likelihood score of two HMM's.

TABLE 1: Changes of the power spectral indices of HRV after task.

\begin{tabular}{|c|c|c|c|c|c|c|c|}
\hline & HR & $\mathrm{LF} / \mathrm{HF}$ & LF (NU) & $\mathrm{LF}(\mathrm{AU})$ & $\mathrm{HF}(\mathrm{NU})$ & $\mathrm{HF}(\mathrm{AU})$ & $\mathrm{TP}$ \\
\hline Pretask & $73.66 \pm 1.38$ & $1.57 \pm 0.21$ & $54.14 \pm 2.46$ & $0.18 \pm 0.03$ & $45.86 \pm 2.46$ & $0.16 \pm 0.02$ & $0.37 \pm 0.05$ \\
\hline Posttask & $69.20 \pm 1.40^{* *}$ & $2.75 \pm 0.36^{* *}$ & $65.40 \pm 2.51^{* *}$ & $0.34 \pm 0.07^{* *}$ & $34.60 \pm 2.51^{* *}$ & $0.17 \pm 0.03$ & $0.56 \pm 0.10^{* *}$ \\
\hline
\end{tabular}

Data is presented as mean \pm SEM. HRV, heart rate variability; HR, heart rate; LF/HF, LF-HF ratio; LF (NU), low frequency in normalized units; LF (AU), low frequency; HF (NU), high frequency in normalized units; HF (AU), high frequency; TP, total power. ${ }^{* *}$ means $P<.005$ versus Pretask.

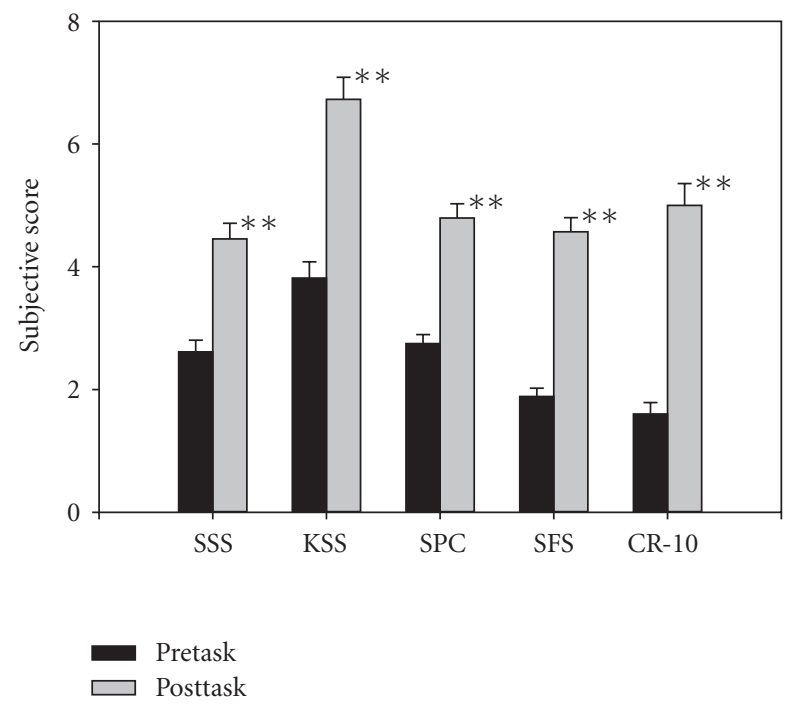

FIGURE 2: Comparison of several subjective scores on mental fatigue between two sessions. Pretask (before task), posttask (immediately after task). SSS, Stanford sleepiness scale; KSS, Karolinska sleepiness scale; SFC, Samn-Perelli checklist; SFS, Li's subjective fatigue scale; CR-10, Borg's CR-10 scale. ${ }^{* *} P<.005$, statistical significance of difference between two sessions.

response time for visual oddball task tends to be prolonged at the posttask measurement, and reaches the significant level $(P<.05)$.The percentage of correctness does not change significantly.

\subsection{Multichannel linear descriptors of EEG}

To eliminate the influences of parameter's fluctuation, the mean value within one minute is calculated to be statistically analyzed. The results of comparison of multichannel linear descriptors in total, $\theta, \alpha$, and $\beta$ frequency bands between two sessions are shown in Figure 6.

Compared with the pretask, mean value of $\Phi$ in total and $\alpha$ frequency band on prefrontal electrodes, central electrodes

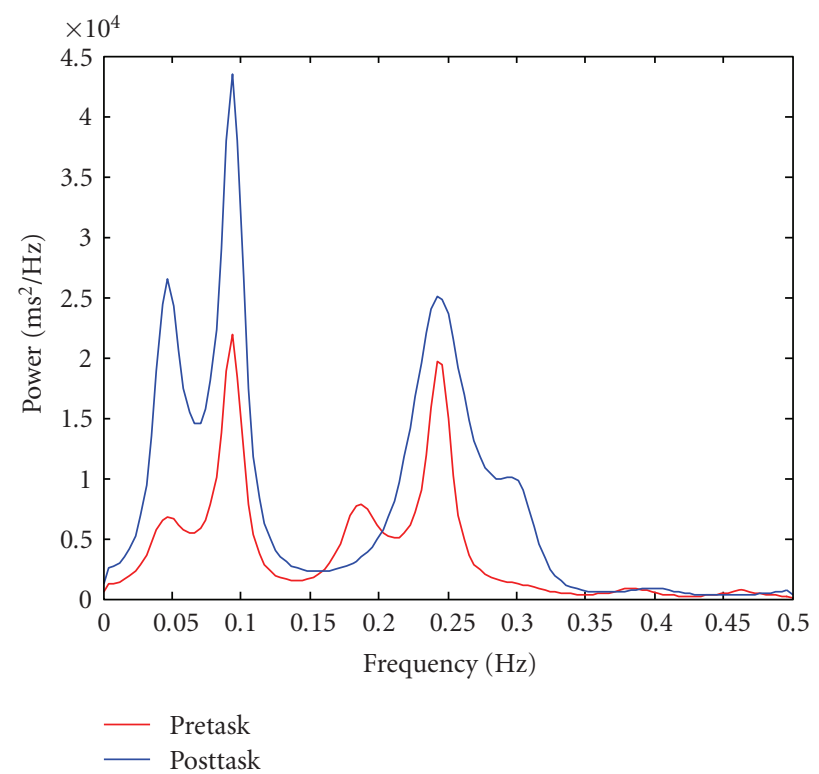

FIgURE 3: Example of power spectral of HRV in the pretask and posttask.

and parietal electrodes, significantly decrease, mean value of $\Omega$ in $\beta$ frequency band on central electrodes and parietal electrodes significantly decrease, while mean value of $\Sigma$ in total, $\beta$ and $\theta$ frequency bands on central electrodes and parietal electrodes significantly increase after the completion of the task. The results indicate that the multichannel linear descriptor features of EEG are closely related with mental fatigue.

\subsection{The classification results by KPCA-HMM}

The subjective measure, power spectrum analysis of HRV and ERP show that the levels of both subjective sleepiness and fatigue increase significantly after long-term VDT task. The subjects are not fatigue and sleepy before task, corresponds to a normal arousal state, and moderately to 




(a)

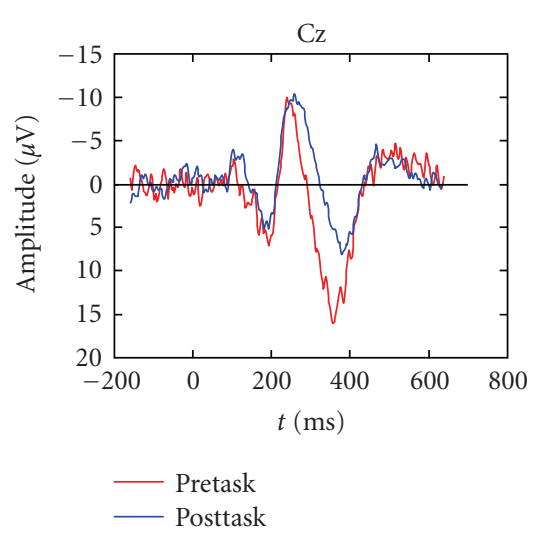

(b)

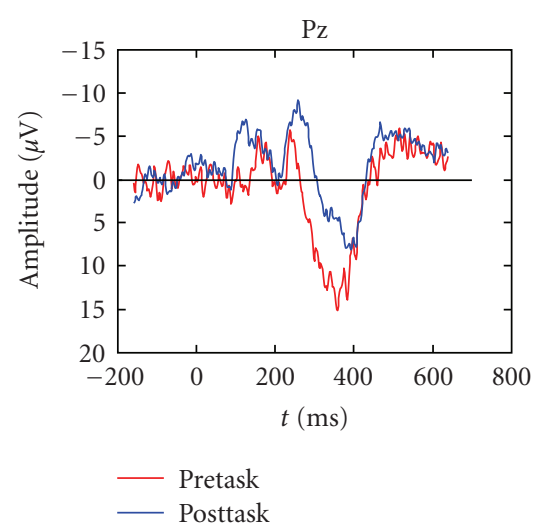

(c)

Figure 4: Examples of P300 waveform at electrode locations Fz, $\mathrm{Cz}$, and Pz in the pretask and posttask.

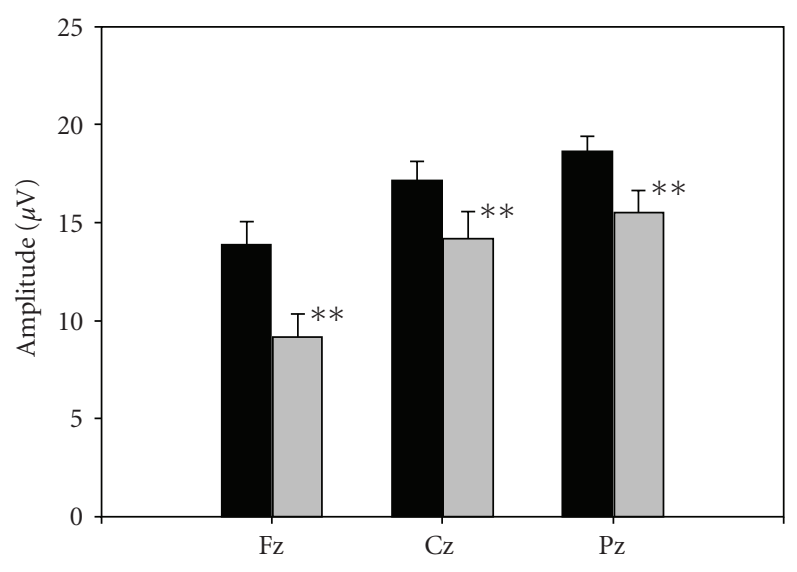

Pretask
$\square$ Posttask



Pretask
Posttask

(a)

(b)

Figure 5: Comparison of P300 amplitude and latency between two sessions. Pretask (before task), posttask (immediately after task). ${ }^{* *} P<$ .005 , statistical significance of difference between two sessions.

extremely fatigue and sleepy after task. In order to differentiate the normal state from the fatigue state, KPCA-HMM is applied. The classification accuracy is observed under the condition of the various extraction features using KPCA and LPCA, respectively. The average classification accuracies for three different HMM's are shown in Figure 7.

Figure 7 illustrates that the accuracy varies with the different number of the feature dimensions. When the dimensionality is more than 15, KPCA-HMM shows a better performance than that of HMM without using KPCA (original HMM). The maximal classification accuracy $(88 \%)$ is reached while the number of feature dimensions equals to 29 , whereas the classification accuracy of original HMM is $86 \%$. The performance of KPCA-HMM is much better than that of LPCA-HMM.

Table 2 shows the comparison of performances when the different physiological parameters are used. The normality of the P300 and HRV parameters and the equality of the corre- sponding covariance matrices are investigated by using onesample Kolmogorov-Smirnov tests and Box's M tests, respectively, before linear-discriminant analysis. The results show that all P300 and HRV parameters meet normality criteria and model covariance matrices are equal.

According to the records, the P300 amplitude and latency of ERP and power spectral of HRV cannot differentiate mental fatigue. Their classification accuracies of mental fatigue are below $65 \%$. However, the performance of KPCA-HMM based on multichannel linear descriptor parameters of EEG is shown to classify mental fatigue effectively, which achieves the maximum recognition accuracy of $88 \%$. Moreover, we observe that the Ac, Sp, and Se of KPCA-HMM are greatly higher than that of the linear-discriminant analysis based on Mahalanobis distance (MDBC) and other HMM methods. This demonstrates KPCA-HMM based on multichannel linear descriptor parameters of EEG is a useful method for detecting the mental fatigue. 


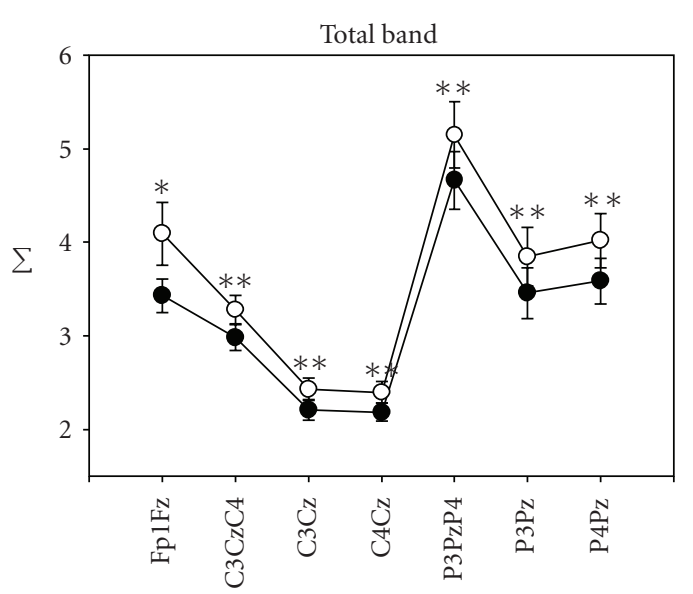

(a)

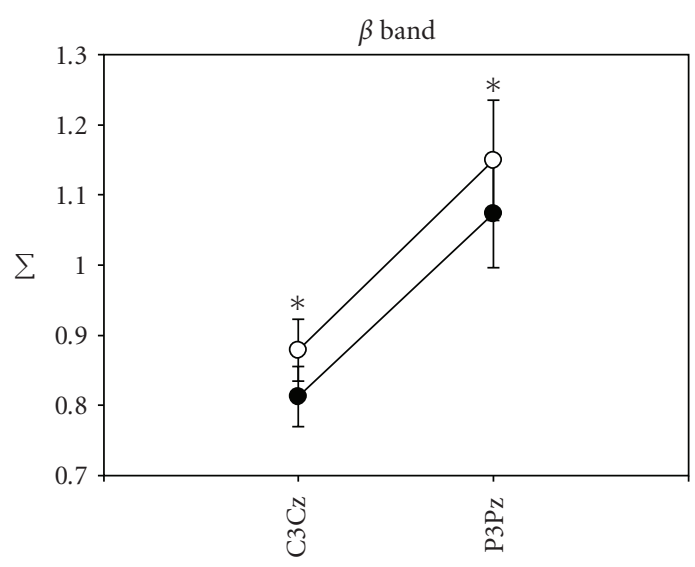

(c)

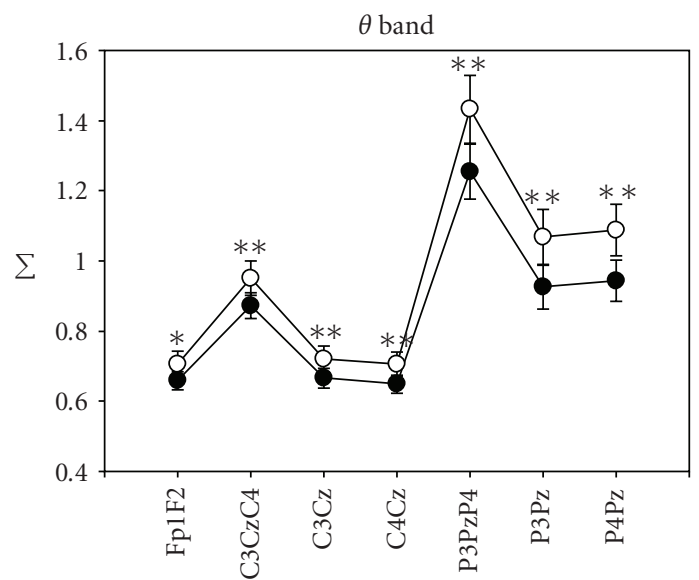

- Pretask

-0 Posttask

(e)



(b)

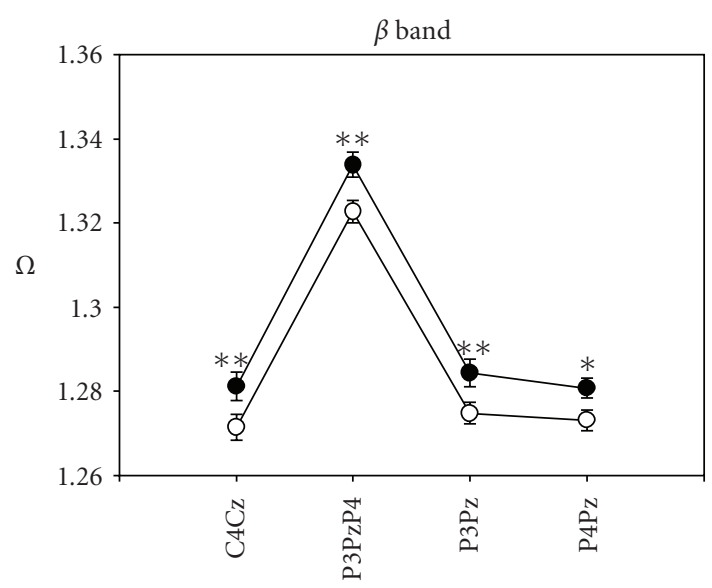

(d)

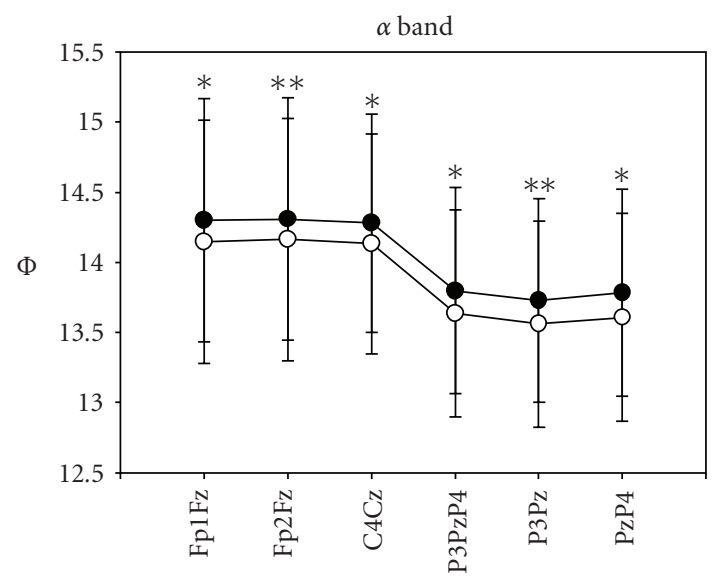

$\longrightarrow$ Pretask

-O- Posttask

(f)

Figure 6: Comparison of multichannel linear descriptors in total, $\beta, \alpha$, and $\theta$ frequency bands between two sessions. Pretask (before task), posttask (immediately after task). ${ }^{*} P<.05,{ }^{* *} P<.005$, statistical significance of difference between two sessions. 
TABLE 2: The comparison of the performances as different physiological parameters are used.

\begin{tabular}{|c|c|c|c|}
\hline Physiological parameters & Techniques & $(\mathrm{Ac}, \mathrm{Sp}, \mathrm{Se})$ & $n$ \\
\hline \multirow{4}{*}{ Multichannel linear descriptor parameters of EEG } & HMM & $(0.86,0.87,0.84)$ & - \\
\hline & КРСА-НMM & $(0.88,0.88,0.88)$ & 29 \\
\hline & LPCA-HMM & $(0.58,0.56,0.59)$ & 17 \\
\hline & MDBC & $(0.67,0.60,0.74)$ & - \\
\hline \multirow{2}{*}{ P300 amplitude and latency of ERP } & HMM & $(0.64,0.68,0.60)$ & - \\
\hline & MDBC & $(0.54,0.78,0.30)$ & - \\
\hline \multirow{2}{*}{ Power spectral indices of HRV } & HMM & $(0.60,0.58,0.62)$ & - \\
\hline & MDBC & $(0.62,0.70,0.54)$ & - \\
\hline
\end{tabular}

$\mathrm{MDBC}$ is the linear discriminant analysis based on Mahalanobis distance; $n$ is the number of principle components; the kernel function of KPCA is RBF function.

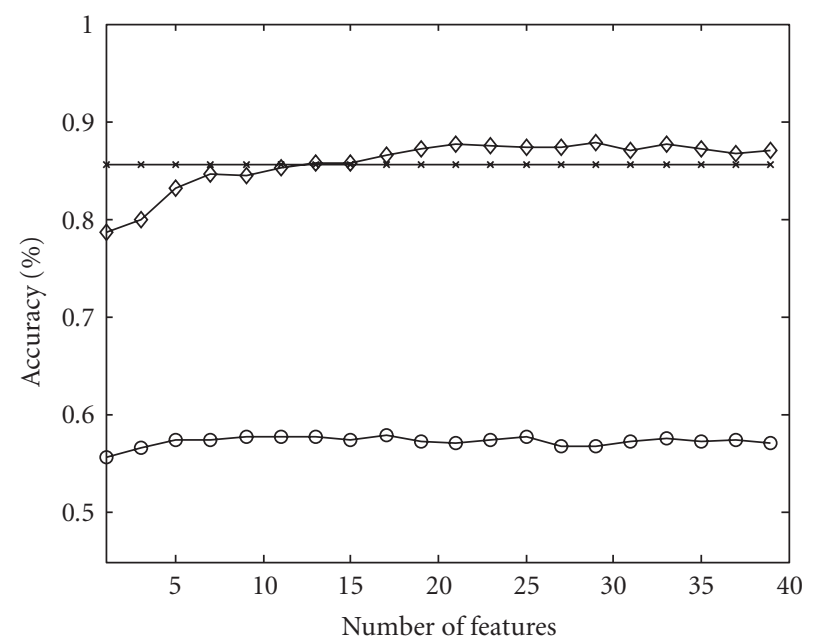

$\begin{array}{ll}\prec & \text { KPCA-HMM } \\ \smile-\text { LPCA-HMM } \\ \rightarrow-\text { Original-HMM }\end{array}$

Figure 7: The average classification accuracies.

\section{DISCUSSIONS AND CONCLUSIONS}

As already mentioned in the introduction, there is a strong link betweenmental fatigue and the autonomic nervous activity, performing monotonous tasks is related to the increase of the LF component in HRV. LF power spectrum of the HRV reflects both sympathetic and parasympathetic activities $[7,22]$. In the present study, there is significant increase in the LF power (AU and NU), LF/HF ratio, and TP power; and a significant decrease in the HF power (NU) after the task compared to that of the pretask period. This is consistent with the analysis of theory and previous research in drive fatigue [7]. Heart is controlled by both sympathetic and parasympathetic activities. When subject is at ease, it is modulated by sympathyovagal balance; on the contrary, the sympathetic activity is predominant when subject is fatigued, excited, and nervous. Therefore, the predominant activity of autonomic nervous system of subjects turns to the sympathetic activity from parasympathetic activity after the task. However, heart rate shows the opposite course as is expected. Continuously, tasking time does not lead heart rate to in- crease. This is in line with the course of the LF component. Lower heart rate causes more variations in heart rate and this is reflected in an increase of the LF component.

The P300 components are useful to measure the ability of cognitive information processing $[15,35]$. It has been reported that the P300 amplitude reflects the depth or degree of cognitively processing the stimulus. In other words, it is strongly related to the level of attention. The P300 latency is found to reflect the temporal aspect when cognitively processing the stimulus. When a cognitive task is difficult to process, the P300 latency will be prolonged. The experimental results show that the P300 amplitudes decrease significantly, while the P300 latencies increase significantly after task, which indicate that the cognitive information processing is slowdown and the cognitive activity will be decreased.

$\Omega$ complexity measures the spatial complexity of brain region and indicates the degree of synchronization between functional processes spatially distributed over different brain regions; $\Phi$ reflects mean frequency of the corresponding field changes; $\Sigma$ describes the field strength of brain region. Moreover, it has been recognized that $\theta$ waves are associated with a variety of psychological states including hypnagogic imagery, low levels of alertness during drowsiness and sleep and as such have been associated with decreased information processing [10], $\alpha$ waves occur during relaxed conditions, at decreased attention levels and in a drowsy but wakeful state, and $\beta$ waves are related to alertness level, and as the activity of $\beta$ band increases, performance of a vigilance task also increases [11]. In our experiment, mean value of $\Omega$ in $\beta$ frequency band on central electrodes and parietal electrodes significantly decreases after task, which suggests the synchronization of the central and parietal cortex increases. Mean value of $\Phi$ in total and $\alpha$ frequency band on prefrontal electrodes, central electrodes and parietal electrodes significantly decreases after task, which reflects the decrease of field changes in prefrontal, central, and parietal brain region. Mean value of $\Sigma$ in total, $\beta$, and $\theta$ frequency bands on central electrodes and parietal electrodes significantly increases after the completion of the task, which reflects the positive change of energy of in total, $\beta$, and $\theta$ frequency bands on central and parietal cortex. The results indicate that the multichannel linear descriptors of EEG can effectively reflect the changes of $\theta$, $\alpha, \beta$ waves, and the change of mental fatigue further. 
HMM shows better performance than that of traditional linear discriminant analysis. The KPCA-HMM by using the multichannel linear descriptor parameters of EEG shows better performance than that by using other physiological parameters. The average recognition accuracy by KPCA-HMM can reach to $88 \%$. It is much better than that by using other models for distinguishing the VDT mental fatigue. Hence KPCA-HMM is a suitable promising method for the mental fatigue estimation.

Long-term VDT task has significant effect on psychology, behaviour and physiology of subjects, which induces the changes of subjective sleepiness and mental fatigue, autonomic nervous function, and central nervous system. In this paper, we focus on the use of physiological methods to measure mental fatigue. The indices based on central nervous system (EEG) and those based on autonomic nervous function such as HRV are combined to monitor the change of mental fatigue. There is a close relationship between changes in fatigue and the physiological parameters. These physiological parameters may serve as indicators of the level of mental fatigue. For mental fatigue classification, experimental study suggests that the joint KPCA-HMM method might be a useful tool in the estimation of mental fatigue, which can effectively reduce the dimensions of the feature vectors, accelerate the classification speed, and improve the accuracy of mental fatigue.

\section{ACKNOWLEDGMENTS}

This work is supported by The National Science Foundation of China under Ggrant no. 30670534. The authors would like to thank the students who kindly participated in this study.

\section{REFERENCES}

[1] S. Konz, "Work/rest: part II-the scientific basis (knowledge base) for the guide," International Journal of Industrial Ergonomics, vol. 22, no. 1, pp. 73-99, 1998.

[2] K. Baker, J. Olson, and D. Morisseau, "Work practices, fatigue, and nuclear power plant safety performance," Human Factors, vol. 36, no. 2, pp. 244-257, 1994.

[3] S. K. L. Lal, R. J. Henderson, N. Carter, et al., "Effect of feedback signal and psychological characteristics on blood pressure self-manipulation capability," Psychophysiology, vol. 35, no. 4, pp. 405-412, 1998.

[4] W. Heller, J. B. Nitschke, M. A. Etienne, and G. A. Miller, "Patterns of regional brain activity differentiate types of anxiety," Journal of Abnormal Psychology, vol. 106, no. 3, pp. 376-385, 1997.

[5] S. K. L. Lal and A. Craig, "Driver fatigue: electroencephalography and psychological assessment," Psychophysiology, vol. 39, no. 3, pp. 313-321, 2002.

[6] N. Egelund, "Spectral analysis of heart rate variability as an indicator of driver fatigue," Ergonomics, vol. 25, no. 7, pp. 663672, 1982.

[7] Z. Li, K. Jiao, M. Chen, and C. Wang, "Effect of magnitopuncture on sympathetic and parasympathetic nerve activities in healthy driver-assessment by power spectrum analysis of heart rate variability," European Journal of Applied Physiology, vol. 88, no. 4-5, pp. 404-410, 2003.

[8] S. K. L. Lal and A. Craig, "A critical review of the psychophysiology of driver fatigue," Biological Psychology, vol. 55, no. 3, pp. 173-194, 2001.

[9] S. K. L. Lal and A. Craig, "Electroencephalography activity associated with driver fatigue: implications for a fatigue countermeasure device," Journal of Psychophysiology, vol. 15, no. 3, pp. 183-189, 2001.

[10] W. Klimesch, "EEG alpha and theta oscillations reflect cognitive and memory performance: a review and analysis," Brain Research Reviews, vol. 29, no. 2-3, pp. 169-195, 1999.

[11] M. Scerbo, F. G. Freeman, and P. J. Mikulka, "A biocybernetic system for adaptive automation," in Engineering Psychophysiology: Issues and Applications, pp. 241-253, Lawrence Erlbaum Associates, Mahwah, NJ, USA, 2000.

[12] H. J. Eoh, M. K. Chung, and S.-H. Kim, "Electroencephalographic study of drowsiness in simulated driving with sleep deprivation," International Journal of Industrial Ergonomics, vol. 35, no. 4, pp. 307-320, 2005.

[13] J. Polich and A. Kok, "Cognitive and biological determinants of P300: an integrative review," Biological Psychology, vol. 41, no. 2, pp. 103-146, 1995.

[14] M. A. S. Boksem, T. F. Meijman, and M. M. Lorist, "Effects of mental fatigue on attention: an ERP study," Cognitive Brain Research, vol. 25, no. 1, pp. 107-116, 2005.

[15] A. Murata, A. Uetake, and Y. Takasawa, "Evaluation of mental fatigue using feature parameter extracted from event-related potential," International Journal of Industrial Ergonomics, vol. 35, no. 8, pp. 761-770, 2005.

[16] M. M. Lorist, M. Klein, S. Nieuwenhuis, R. De Jong, G. Mulder, and T. F. Meijman, "Mental fatigue and task control: planning and preparation," Psychophysiology, vol. 37, no. 5, pp. 614-625, 2000.

[17] E. Hoddes, V. Zarcone, H. Smythe, R. Phillips, and W. C. Dement, "Quantification of sleepiness: a new approach," Psychophysiology, vol. 10, no. 4, pp. 431-436, 1973.

[18] T. Akerstedt and M. Gillberg, "Subjective and objective sleepiness in the active individual," International Journal of Neuroscience, vol. 52, no. 1-2, pp. 29-37, 1990.

[19] S. W. Samn and L. P. Perelli, "Estimating aircrew fatigue: a technique with implications to airlift operations," Tech. Rep. SAM-TR-82-21, USAF School of Aerospace Medicine, Brooks AFB, Tex, USA, 1982.

[20] G. Borg, Borg's Perceived Exertion and Pain Scales, Human Kinetics, Champaign, Ill, USA, 1998.

[21] T. Miyashita, K. Ogawa, H. Itoh, et al., "Spectral analyses of electroencephalography and heart rate variability during sleep in normal subjects," Autonomic Neuroscience, vol. 103, no. 1-2, pp. 114-120, 2003.

[22] M. Pagani, F. Lombardi, S. Guzzetti, et al., "Power spectral analysis of heart rate and arterial pressure variabilities as a marker of sympathovagal interaction in man and conscious dog," Circulation Research, vol. 58, pp. 178-193, 1986.

[23] N. Montano, T. G. Ruscone, A. Porta, F. Lombardi, M. Pagani, and A. Malliani, "Power spectrum analysis of heart rate variability to assess the changes in sympathovagal balance during graded orthostatic tilt," Circulation, vol. 90, no. 4, pp. 1826$1831,1994$.

[24] B. Pomeranz, R. J. B. MacAuley, M. A. Caudill, et al., "Assessment of autonomic function in humans by heart rate spectral analysis," American Journal of Physiology, vol. 248, no. 1, pp. H151-H153, 1985. 
[25] J. Wackermann, "Towards a quantitative characterization of functional states of the brain: from the non-linear methodology to the global linear description," International Journal of Psychophysiology, vol. 34, no. 1, pp. 65-80, 1999.

[26] X. M. Pei, C. Zheng, A. H. Zhang, F. J. Duan, and G. Y. Bin, "Discussion on "towards a quantitative characterization of functional states of the brain: from the non-linear methodology to the global linear description" by J. Wackermann," International Journal of Psychophysiology, vol. 56, no. 3, pp. 201207, 2005.

[27] B. Schölkopf and A. J. Smola, Learning with Kernels: Support Vector Machines, Regularization, Optimization and Beyond, MIT Press, Cambridge, Mass, USA, 2002.

[28] K.-R. Muller, S. Mika, G. Ratsch, K. Tsuda, and B. Scholkopf, "An introduction to kernel-based learning algorithms," IEEE Transactions on Neural Networks, vol. 12, no. 2, pp. 181-201, 2001.

[29] R. Rosipal, M. Girolami, L. J. Trejo, and A. Cichocki, "Kernel PCA for feature extraction and de-noising in nonlinear regression," Neural Computing \& Applications, vol. 10, no. 3, pp. 231-243, 2001.

[30] A. P. Dempster, N. M. Laird, and D. B. Rubin, "Maximum likelihood from incomplete data via the EM algorithm," Journal of the Royal Statistical Society, vol. 39, no. 1, pp. 1-38, 1977.

[31] L. Rabiner and B. H. Juang, Fundamentals of Speech Recognition, Prentice-Hall, Englewood Cliffs, NJ, USA, 1993.

[32] C. H. Q. Ding and I. Dubchak, "Multi-class protein fold recognition using support vector machines and neural networks," Bioinformatics, vol. 17, no. 4, pp. 349-358, 2001.

[33] S. Theodoridis and K. Koutroumbas, Pattern Recognition, Academic Press, New York, NY, USA, 2nd edition, 2003.

[34] C. C. C. Pang, A. R. M. Upton, G. Shine, and M. V. Kamath, "A comparison of algorithms for detection of spikes in the electroencephalogram," IEEE Transactions on Biomedical Engineering, vol. 50, no. 4, pp. 521-526, 2003.

[35] A. Uetake and A. Murata, "Assessment of mental fatigue during VDT task using event-related potential (P300)," in Proceedings of the 9th IEEE International Workshop on Robot and Human Interactive Communication (RO-MAN '00), pp. 235240, Osaka, Japan, September 2000. 